\title{
Pharmacokinetics of methylphenidate and ritalinic acid in plasma correlations with exhaled breath and oral fluid in healthy volunteers
}

\author{
Michel Arvidsson ${ }^{1}$ (i) $\cdot$ Marja-Liisa Dahl ${ }^{1} \cdot$ Olof Beck $^{1} \cdot$ Gerd Ackehed $^{1} \cdot$ Karin Nordin $^{1} \cdot$ Staffan Rosenborg $^{1}$
}

Received: 30 July 2019 / Accepted: 23 October 2019 / Published online: 30 November 2019

(C) The Author(s) 2019

\begin{abstract}
Purpose The primary aim of this study was to explore the potential of alternative sampling matrices for methylphenidate by assessing the correlations between $d l$-threo-methylphenidate and $d l$-threo-ritalinic acid concentrations in exhaled breath and oral fluid with those in plasma, in repeated samples collected after a single oral dose of methylphenidate. The secondary aim was to study the enantioselective pharmacokinetics of methylphenidate in plasma, with a focus on interindividual variability in the metabolism of methylphenidate to ritalinic acid.

Methods Twelve healthy volunteers received a single oral dose of $d l$-threo-methylphenidate (Ritalin ${ }^{\circledR}$ capsules, $20 \mathrm{mg}$ ). Venous blood samples were collected for $24 \mathrm{~h}$, and plasma analyzed for threo-enantiomers of methylphenidate and ritalinic acid with LCMS/MS. Repeated sampling of exhaled breath, using a particle filter device, and of non-stimulated oral fluid, using a felt pad device, was also performed. Exhaled breath and oral fluid were analyzed with a non-enantioselective LC-MS/MS method for $d l$ threo-methylphenidate and dl-threo-ritalinic acid.

Results In all subjects, $d$-threo-methylphenidate was detectable in plasma for at least $15 \mathrm{~h}$ after the dose with a biphasic profile. $l$ threo-Methylphenidate was measurable in only five subjects and in most cases in low concentrations. However, one female subject displayed a biphasic concentration-time profile for l-threo-methylphenidate. This subject also had the highest $d$-threo-methylphenidate AUC (191 ng*h/mL versus 32-119 ng*h/mL in the other subjects). $d$-threo-Ritalinic acid concentrations were on average 25-fold higher (range 6-126) than the corresponding $d$-threo-methylphenidate concentrations. Single-time point plasma concentration ratios between $d$-threo-ritalinic acid and $d$-threo-methylphenidate $1.5-12 \mathrm{~h}$ after dose correlated highly ( $r=0.88-0.98)$ with the $d$-threo-ritalinic acid AUC/d-threo-methylphenidate AUC ratio. In eleven subjects, dl-threo-methylphenidate in oral fluid mirrored the biphasic profile of methylphenidate (sum of $d$-and l-threo-enantiomers) in plasma, but the concentrations in oral fluid were on average 1.8 times higher than in plasma. dl-threo-Methylphenidate was detected in exhaled breath in all subjects, but there was no consistent concentration-time pattern.

Conclusions In some subjects, the pharmacologically less active l-threo-enantiomer may contribute to the total plasma methylphenidate concentrations. Monitoring methylphenidate concentrations without enantiomeric determination carries the risk of missing such subjects, which might affect how the plasma concentrations of methylphenidate are interpreted and used for clinical decision making. The use of exhaled breath and oral fluid to assess medication adherence to MPH in patients with ADHD warrants further studies.
\end{abstract}

Keywords Methylphenidate $\cdot$ Ritalinic acid $\cdot$ Exhaled breath $\cdot$ Oral fluid $\cdot$ Enantioselective pharmacokinetics

Electronic supplementary material The online version of this article (https://doi.org/10.1007/s00228-019-02787-x) contains supplementary material, which is available to authorized users.

Michel Arvidsson

michel.arvidsson@ki.se

1 Clinical Pharmacology, Department of Laboratory Medicine,

Karolinska Institutet, and Karolinska University Laboratory,

Karolinska University Hospital, Huddinge, SE-141

86 Stockholm, Sweden

\section{Introduction}

Attention-deficit hyperactivity disorder (ADHD) is a psychiatric disorder with a worldwide pooled prevalence of $5.29 \%$ $(95 \% \mathrm{CI}=5.01-5.56)$ in children and adolescents and $2.5 \%$ (95\% CI 2.1-3.1) in adults [1, 2]. The first-line pharmacological treatment for ADHD is stimulant medications, either methylphenidate (MPH) or amphetamine [3]. In 2010, methylphenidate was the most commonly prescribed medication 
for ADHD in most countries, except in the US, where amphetamine was most common [4].

Methylphenidate is a chiral compound with four stereoisomers (two pairs of enantiomers), the dextro- and laevo-enantiomers of erythro- and threo-methylphenidate, meaning that four isomers exist: $d$-threo-(2R:2'R)-methylphenidate, $l$ threo-(2S:2'S)-methylphenidate, d-erythro-(2R:2' $S)$-methylphe-

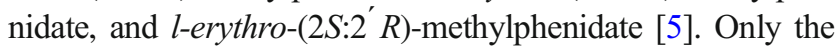
threo pair of enantiomers accounts for the central stimulant effect and a racemic mixture (50:50) of the threo-enantiomers, $d$-threo-(2R:2 $R)$-methylphenidate, hereafter referred to as $d$ threo-methylphenidate and l-threo-(2S:2' $S)$-methylphenidate, hereafter referred to as l-threo-methylphenidate is used in drug therapy $[6,7]$. The main route of methylphenidate metabolism is de-esterification via the enzyme carboxylesterase 1 (CES1) to the inactive metabolite ritalinic acid (RA) [8]. The metabolism of methylphenidate is enantioselective, and the catalytic activity of CES1 is 6-7 times higher for l-threo-methylphenidate versus d-threo-methylphenidate [9]. Patients with deficient CES1 activity have been described to have at least 7-fold higher maximum plasma concentrations of methylphenidate after a single dose of the drug compared with other subjects [10]. The absolute bioavailability of $l$-threo-methylphenidate after oral administration is only $5 \%$ or less compared with $23 \%$ for $d$-threo-methylphenidate, indicating extensive first-pass metabolism of l-threo-methylphenidate by CES1 [8, 11].

The significant interindividual differences in plasma concentrations after similar doses of methylphenidate indicate that the dosage should be individually titrated for optimal effect and to avoid toxicity [6]. The monitoring of methylphenidate concentrations is usually based on non-enantioselective methods [12]. However, as the $d$-threo-enantiomer is considered to be pharmacologically more active than the l-threo-enantiomer [13, 14], the determination of enantiomer concentrations could be crucial. Furthermore, the analysis of both the parent drug and metabolite could be informative concerning individual metabolic capacity. For example, the ratio between risperidone and its primary metabolite 9-hydroxyrisperidone reflects the activity of the cytochrome P450 CYP2D6 $[15,16]$.

Medication adherence rates are low in patients with ADHD [17]. The global increase in the utilization of central stimulant medications (methylphenidate and amphetamine), with the potential of abuse, misuse, and diversion of the drugs, highlights the importance of monitoring medication adherence to minimize these risks but also to optimize prescription [4]. Therapeutic drug monitoring (TDM) is an established concept of quantification and interpretation of drug concentrations in biological fluids (most often in whole blood, plasma, or serum) to guide individualized dosing and for monitoring medication adherence [18, 19]. Over the last decade, there has been a growing interest in alternatives to the traditional sampling matrices, blood, and urine, and significant methodological advances have been made $[20,21]$. Oral fluid specimens are easy-to-collect and can be used when there is a risk of adulteration of urine. Some issues related to the collection of oral fluid are dryness of mouth and contamination from food [22]. The collection of exhaled breath is also non-invasive and easy to perform. However, the low analyte concentrations are methodologically challenging [23], and many factors influencing drug concentrations in exhaled breath are unknown.

The primary aim of this study was to explore the potential use of alternative sampling matrices for adherence and TDM, by assessing the correlations between dl-threo-methylphenidate and dl-threo-ritalinic acid concentrations in exhaled breath and oral fluid with those in plasma, in repeated samples collected after a single oral dose. The secondary aim was to study the enantioselective pharmacokinetics of methylphenidate in plasma, with a focus on interindividual variability in metabolism to ritalinic acid.

\section{Materials and methods}

\section{Study design}

The study was conducted at the Clinical Pharmacology Trial Unit, Karolinska University Hospital, Huddinge, Sweden. The study was not classified as a clinical trial according to the European guidelines [24], as we did not intend to study the effects or safety of methylphenidate. We conducted the study according to the Declaration of Helsinki (Fortaleza, 2013) and the International Conference on Harmonization guidelines for Good Clinical Practice. The Regional Ethics Committee in Stockholm (No. 2016/2297-31) approved the study. Subjects were recruited through the Clinical Pharmacology Trial Unit website, were eligible if they were between 18 and 65 years, and gave informed consent after written and oral information. All subjects enrolled were considered in good health as assessed at screening within 30 days prior to study drug administration if medical history, physical examination, 12-lead electrocardiogram, and routine laboratory tests including complete blood count, serology (hepatitis B, hepatitis C, HIV), serum electrolytes, blood glucose, urine drug screen, and liver function tests were normal. Female subjects were required to use appropriate contraceptive measures throughout the study and to have a negative urine pregnancy test at screening and before study drug administration. All subjects with current or previous known alcohol use disorder or substance use disorder, a current and clinically significant medical, psychiatric, or surgical illness, known hypersensitivity to methylphenidate, or if the investigator considered them unsuitable for the study were excluded. Subjects who participated in another clinical study for the last 3 months were excluded.

The subjects arrived at the trial unit in the morning, $1 \mathrm{~h}$ before receiving the study drug, and were housed at the unit 
for the duration of the study. They were fasting at least $10 \mathrm{~h}$ before dosing and received standardized meals during the study. Breakfast was served $2 \mathrm{~h}$ post-dose. They received a single oral dose of methylphenidate, $20 \mathrm{mg}$, Ritalin ${ }^{\circledR}$ capsules, which is a recommended starting dose for adults with ADHD, and the adverse events are known. This capsule contains methylphenidate formulated with $50 \%$ of the dose as immediate-release beads and $50 \%$ as delayed-release beads, resulting in a bi-modal release profile of methylphenidate. The sampling of venous blood, exhaled breath, and oral fluid was done according to the sampling scheme (Electronic suppl. Table 1). The subjects left the clinical center $1 \mathrm{~h}$ after the last sample was taken at $24 \mathrm{~h}$ after the dose.

\section{Sample preparation}

Venous blood was collected into $3 \mathrm{~mL}$ tubes containing an FC-mixture, consisting of $\mathrm{Na}_{2}$ EDTA, sodium fluoride, citric acid, and sodium citrate (Vacuette, Greiner Bio-One). The samples were centrifuged within $60 \mathrm{~min}(1500 \times \mathrm{g}$ for 10 $\mathrm{min}$ ) and the plasma was transferred into polypropylene tubes (Nunc cryotubes vials, Thermo Scientific) and stored at -70 ${ }^{\circ} \mathrm{C}$ until analysis.

Exhaled breath was collected with a sampling device described earlier [25]. The mouthpiece only allowed microparticles to pass through and be collected on a polymer filter inside the device [26]. After sampling, the device was sealed and stored at $-20^{\circ} \mathrm{C}$. Stability of exhaled breath samples was documented for three months at $-20^{\circ} \mathrm{C}$.

One milliliter of oral fluid was collected using a sampling device (Quantisal, Immunalysis, Pomona, CA, USA) according to the manufacturer's recommendations. The collection pad was placed in $3 \mathrm{~mL}$ of buffer solution (i.e., dilution 1:4). The device was sealed, left at room temperature for at least 6 $\mathrm{h}$, and then stored at $-20^{\circ} \mathrm{C}$.

\section{Enantioselective determination of dl-threo-methylphenidate and dl-threo-ritalinic acid in plasma}

A new enantioselective method was developed and will be described in detail in a separate paper. Briefly, sample preparation was performed by transferring aliquots of $200 \mu \mathrm{L}$ plasma to an Ostro 96-well plate $(25 \mathrm{mg}$, Waters, Milford, MA, USA). Protein precipitation was performed by adding $600 \mu \mathrm{L}$ of internal standard solution $(0.1 \%$ formic acid and $15 \%$ methanol in acetonitrile containing the internal standards $( \pm)$-threomethylphenidate-d4 (MPH-d4) and ( \pm )-threo-ritalinic acid-d4 (ritalinic acid-d4); Cerilliant, Round Rock TX, USA). After thorough mixing, the Ostro-plate was placed above a $1 \mathrm{~mL}$ 96deep well plate (Nunc PP plate, Thermo Scientific) and centrifuged for $5 \mathrm{~min}$ at $22^{\circ} \mathrm{C}$ and $3400 \mathrm{rpm}$ (Heraeus Megafuge 1.0). The filtrates were evaporated to dryness and resolved in
$50 \mu \mathrm{L}$ of $10 \mathrm{mmol} / \mathrm{L}$ ammonium formate, $\mathrm{pH} 5.35 \pm 0.05$, mixed for $5 \mathrm{~min}$ and centrifuged for $5 \mathrm{~min}$ at $4{ }^{\circ} \mathrm{C}$ and 3400 rpm.

The samples were analyzed on an LC-MS/MS system (TSQ Quantiva with Dionex Ultimate 3000 system, Thermo Scientific, Waltham, MA, USA). The analytical column used was a Chiralpak AGP HPLC Column $(100 \times 2.0 \mathrm{~mm}, 5 \mu \mathrm{m}$, Chiral Technologies, West Chester, PA, USA). The chromatographic system was using $10 \mathrm{mmol} / \mathrm{L}$ ammonium formate adjusted with formic acid to $\mathrm{pH} 5.35 \pm 0.05$ as mobile phase $\mathrm{A}$ and $100 \%$ methanol as mobile phase $\mathrm{B}$. The proportion of mobile phase $\mathrm{B}$ in the isocratic setting was $4 \%$. The flow rate was $0.250 \mathrm{~mL} / \mathrm{min}$, and the injection volume was $5 \mu \mathrm{L}$. Tandem mass spectrometry (MS/MS) detection was conducted by electrospray ionization in the positive ion mode by monitoring the following precursor/fragment ions: $\mathrm{m} / \mathrm{z} 234.2$ $>84.2 .2$ (dl-threo-methylphenidate), $\mathrm{m} / \mathrm{z} 238.2>88.1$ (internal standard methylphenidate-d4), $m / z, 234.2>84.1$ (dl-threoritalinic acid), and $\mathrm{m} / \mathrm{z} 224.2>88.1$ (internal standard ritalinic acid-d4). The analytical method was validated in the concentration range 0.050 to $20.0 \mathrm{ng} / \mathrm{mL}$ for $d$ - and l-threo-methylphenidate and in the range 0.150 to $60.0 \mathrm{ng} / \mathrm{mL}$ for $d$ - and $l$ threo-ritalinic acid. The validation was performed according to the guideline on bioanalytical method validation (EMEA/CHMP/EWP/192217/2009 Rev.1 Corr.2). The results were successfully fulfilling all the criteria of the guideline. The precision reported as the CV for the different enantiomers varied between 3.7 and $10.5 \%$ at the lower limit of quantification (LLOQ) and between 2.7 and $7.1 \%$ for all other quality-control (QC) levels. The accuracy reported as the bias for the different enantiomers varied between 9.0 and $17.6 \%$ at the LLOQ level and between -5.0 and $2.7 \%$ for all other QC levels.

\section{Analysis of dl-threo-methylphenidate and $\mathrm{dl}$-threo-ritalinic acid in exhaled breath and oral fluid}

The exhaled breath samples were analyzed with a previously published method [27]. In brief, the exhaled breath devices were put on top of glass test tubes, and analytes were eluted from the prewetted filter with methanol avoiding contact with the inner walls of the device. After evaporation to dryness, the extract was reconstituted in $80 \mu \mathrm{L}$ of methanol/water $(1: 1$, $\mathrm{v} / \mathrm{v}$ ) and $2 \mu \mathrm{L}$ injected into the LC-MS/MS system.

One hundred microliters of the oral fluid /buffer sample was diluted with $25 \mu \mathrm{L}$ of internal standard solution (methylphenidate-d4 and ritalinic acid-d4 in water) and $200 \mu \mathrm{L}$ of a mixture of methanol/acetonitrile $(1: 1, \mathrm{v} / \mathrm{v})$. After shaking and centrifugation, the supernatant was transferred into glass vials and $2 \mu \mathrm{L}$ injected into the LC-MS/MS system, with the same instrumental settings as for exhaled breath [23]. The concentration in the neat oral fluid was calculated from the measured 
concentration in the buffer multiplied by 4 to compensate for the dilution in the first step of sample handling (see 2.2).

The lower limits of quantification for methylphenidate were $7 \mathrm{pg} /$ filter in exhaled breath and $0.7 \mathrm{ng} / \mathrm{mL}$ in oral fluid and for ritalinic acid $4 \mathrm{pg} /$ filter in exhaled breath and $0.2 \mathrm{ng} /$ $\mathrm{mL}$ in oral fluid.

\subsection{Pharmacokinetic calculations and statistical analysis}

Non-compartmental pharmacokinetic parameters were calculated using PKSolver 2.0 [28] for Microsoft Excel. From plasma concentrations of the enantiomers of methylphenidate and ritalinic acid, the following parameters were determined: peak concentration $\left(\mathrm{C}_{\max }\right)$, time to reach peak concentration $\left(\mathrm{t}_{\max }\right)$, the area under the concentration-time curve $\left(\mathrm{AUC}_{\text {inf_obs }}\right)$, elimination half-life $\left(\mathrm{t}_{1} / 2\right)$, and oral clearance $\left(\mathrm{Cl} / \mathrm{F}_{\mathrm{obs}}\right)$. The ratio between $d$-threo-ritalinic acid and $d$-threo-methylphenidate (d-RA/d-MPH) in plasma at different time points and that between the AUCs of $d$-threo-ritalinic acid and d-threo-methylphenidate $\left(\mathrm{AUC}_{\mathrm{d}-\mathrm{RA}} / \mathrm{AUC}_{\mathrm{d}-\mathrm{MPH}}\right)$ were calculated and correlations between them expressed by Pearson correlation coefficients. Linear regression was used for comparison between matrices. Comparisons between men and women were calculated with Mann-Whitney $U$ test. GraphPad Prism 7 (GraphPad Software, Inc., San Diego, CA, USA) was used for drawing graphs and making statistical analyses.

\section{Results}

Thirteen subjects were screened, and twelve subjects were eligible for the study (six women and six men). They were Caucasian with a mean age of 29.5 years (range, 22-42 years) and a mean weight for women of $63 \mathrm{~kg}$ (range, $57-76 \mathrm{~kg}$ ) and for men of $81 \mathrm{~kg}$ (range, 68-97 kg). The mean BMI for women was 22.4 (range, 19.6-27.8) and for men 25.1 (range, 22-29.9). Five adverse events were reported, four of which were related to ongoing mild upper respiratory tract infection present before dosing of methylphenidate. Only one subject reported adverse events (mild lightheadedness) during the study day.

Blood samples were collected at 18 time points, including the pre-dose sample. l-threo-Methylphenidate was measurable in only five subjects and in most cases, in low concentrations. As expected, due to the galenic profile of the extended-release Ritalin ${ }^{\circledR}$ capsules, with both immediate-release and delayedrelease beads, plasma concentrations of $d$-threo-methylphenidate displayed a biphasic profile in all subjects with mean $\mathrm{t}_{\max 1}$ of $2 \mathrm{~h}$ (range $0.5-3 \mathrm{~h}$ ) and $\mathrm{t}_{\max 2}$ of $5 \mathrm{~h}$ (range 3-10 h). The main pharmacokinetic parameters in plasma are presented in Electronic suppl. Tables 2 and 3.

Both $d$ - and l-threo-ritalinic acid plasma concentrations were quantifiable in all post-dose samples. $d$-threo-Ritalinic acid concentrations were higher (mean 25-fold, range 6-126fold; $N=204$ ) than the corresponding $d$-threo-methylphenidate concentrations. Similarly, l-threo-ritalinic acid concentrations were much higher (mean 315-fold; range 5-1614-fold; $N$ $=32$ ) than the l-threo-methylphenidate concentrations in the five subjects where both were measurable. The individual concentration-time curves for $d$ - and l-threo-methylphenidate and $d$ - and $l$-threo-ritalinic acid in plasma are shown in Fig. 1.

One female subject (subject 9) displayed an unusual concentration-time profile in plasma for both $d$ - and l-threomethylphenidate, Fig. 2. She had the highest $d$-threo-methylphenidate exposure of all subjects with an AUC 0-inf ${ }_{\text {obs }}$ of $191 \mathrm{ng} * \mathrm{~h} / \mathrm{mL}$ (range $32-119 \mathrm{ng} * \mathrm{~h} / \mathrm{mL}$ in the other subjects) (Electronic suppl. Table 2). l-threo-Methylphenidate was measurable in 15 out of 17 samples in this outlier and showed a biphasic profile, Fig 1 . She also displayed the longest $d$-threomethylphenidate $\mathrm{t}_{1 / 2}(4.5 \mathrm{~h})$ and the lowest $d$ - and l-threoritalinic acid $\mathrm{C}_{\max }$ of all study subjects. The clearance of $d$ threo-methylphenidate in this female outlier was low (105 $\mathrm{mL} / \mathrm{h}$ ) compared with the other participants (range 168-626 $\mathrm{mL} / \mathrm{h}$ ), and the $\mathrm{AUC}_{\mathrm{d}-\mathrm{RA}} / \mathrm{AUC}_{\mathrm{d}-\mathrm{MPH}}$ ratio was $70 \%$ lower [7] compared with the other eleven subjects (range 13-40) (Electronic suppl. Table 2).

The Pearson correlation coefficients between individual d$\mathrm{RA} / \mathrm{d}-\mathrm{MPH}$ concentration ratios at different time points versus the $\mathrm{AUC}_{\mathrm{d}-\mathrm{RA}} / \mathrm{AUC}_{\mathrm{d}-\mathrm{MPH}}$ ratios were determined and plotted against the time, Fig. 3. The best correlations were found for samples taken 4-12 h ( $r=0.94-0.98)$ after the dose. However, $1.5-3 \mathrm{~h}$ after the dose, the correlations were already relatively high, $r=0.88$. The ratios for the samples taken $5 \mathrm{~h}(r=0.98)$ after the dose are shown in Fig. 4.

Women tended to exhibit higher $d$-threo-methylphenidate plasma concentrations compared with men. The median $d$ threo-methylphenidate AUC was $73 \mathrm{ng} * \mathrm{~h} / \mathrm{mL}$ (interquartile range, IQR 46-137 $\mathrm{ng} * \mathrm{~h} / \mathrm{mL}$ ) for women and $55 \mathrm{ng} * \mathrm{~h} / \mathrm{mL}$ (IQR $25-66 \mathrm{ng} * \mathrm{~h} / \mathrm{mL}$ ) for men. However, the difference was not statistically significant $(p=0.31)$.

dl-threo-Methylphenidate was detected in all oral fluid samples up to $8 \mathrm{~h}$ and in many samples, up to $12 \mathrm{~h}$ or longer (Electronic suppl. Fig. S1). In eleven subjects, dl-threo-methylphenidate in oral fluid mirrored the biphasic profile of methylphenidate (sum of $d$ - and l-threo-enantiomers) in plasma (Electronic suppl. Fig. S1). The correlation between oral fluid and plasma methylphenidate concentrations $(r=0.61)$ is shown in Fig. 5. The median oral fluid to plasma ratio of $d l$ threo-methylphenidate was 1.8 (range $0.2-14, N=103$ ). In contrast, dl-threo-ritalinic acid concentrations were higher (median ratio 32, range 4-110) in plasma than in oral fluid in all subjects (data not shown).

dl-threo-Methylphenidate was detectable in $87 \%$ (146) of all 168 exhaled breath samples. In five subjects, dl-threomethylphenidate was detected in all samples up to $24 \mathrm{~h}$ after the dose. However, there was no consistent concentration- 

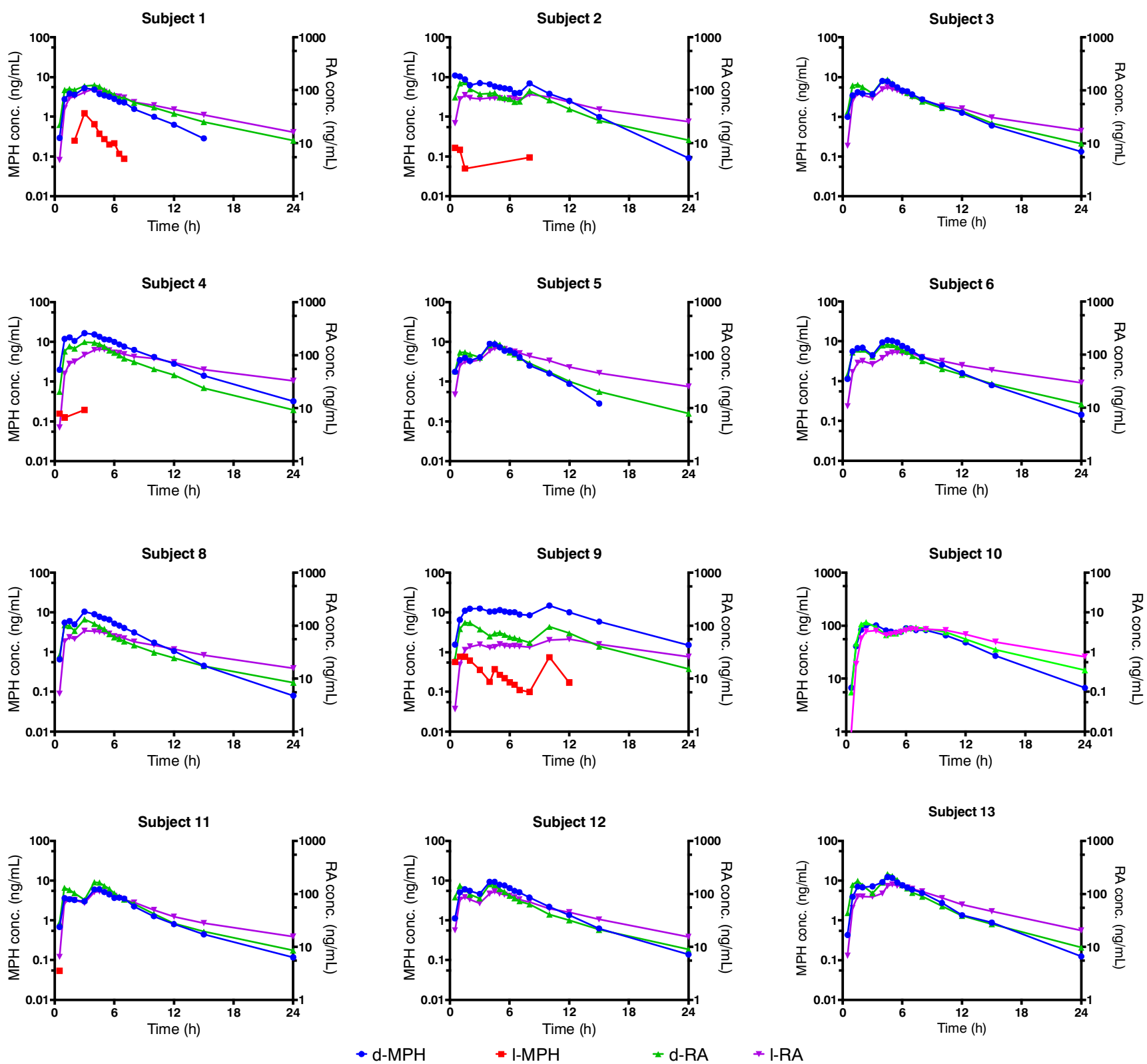

Fig. 1 Individual concentration-time profiles of d- and 1-threo-methylphenidate (MPH) and d- and 1-threo-ritalinic acid (RA) in plasma

time pattern. dl-threo-Ritalinic acid was detectable in only $1 \%$ $(2 / 168)$ of the exhaled breath samples.

\section{Discussion}

There are several key findings from this pharmacokinetic study in healthy volunteers assessing the correlation of methylphenidate concentrations in exhaled breath and oral fluid with those in plasma. The results indicate that monitoring racemic methylphenidate plasma concentrations without recognizing stereoselectivity carries the risk of missing subjects with unusually high concentrations of the pharmacologically less active l-threo-methylphenidate, thus contributing more than expected to the total $d l$-threo-methylphenidate concentration. This might affect how the plasma concentrations of methylphenidate are interpreted and used for clinical decision-making.

Thomsen et al. [29] suggested that a low ratio of $d$ threo- to l-threo-methylphenidate plasma concentration might identify a subject as a poor metabolizer of methylphenidate via CES1. In the present single-dose study, the plasma concentrations of l-threo-methylphenidate were detectable in only five of the twelve subjects and even then in only a minority of the samples. This ratio is therefore unlikely to be useful in a clinical setting. There are 


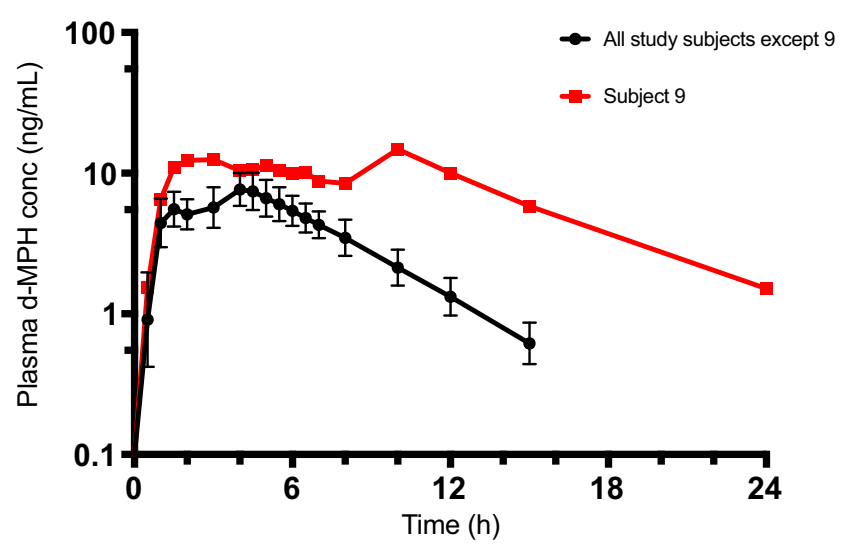

Fig. 2 The plasma concentration versus time curve presenting individual d-threo-methylphenidate (d-MPH) concentrations in the outlier compared with the geometric mean values $( \pm 95 \% \mathrm{CI})$ of 11 study peers following a single $20 \mathrm{mg}$ dose of Ritalin ${ }^{\circledR}$

many examples of the successful use of the ratio between a metabolite and its parent drug (or the opposite) as a measure of enzyme activity, for assessment of inter- and intraindividual variability in the metabolic capacity of specific enzymes. One example is the risperidone/9hydroxyrisperidone ratio, shown to correlate with CYP2D6 activity and to predict extreme genotypes of CYP2D6 [15, 16]. AUC ratios would be scientifically optimal but not practically feasible for clinical use as they require multiple sampling. For TDM of methylphenidate, sampling of plasma at $1-2 \mathrm{~h}$ after immediate release or between 5 and $8 \mathrm{~h}$ for extended-release formulations are recommended [19]. Stage et al. [30] suggested the ritalinic acid/methylphenidate metabolic ratio based on a singlepoint measurement, $3 \mathrm{~h}$ after dose $\left(10 \mathrm{mg}\right.$, Ritalin $\left.{ }^{\circledR}\right)$ as a marker of CES1 activity. The time point was chosen because of a high correlation with the corresponding AUC ratios $(r \geq 0.90)$ from $3 \mathrm{~h}$ and onwards. One single-point measurement instead of repeated sampling

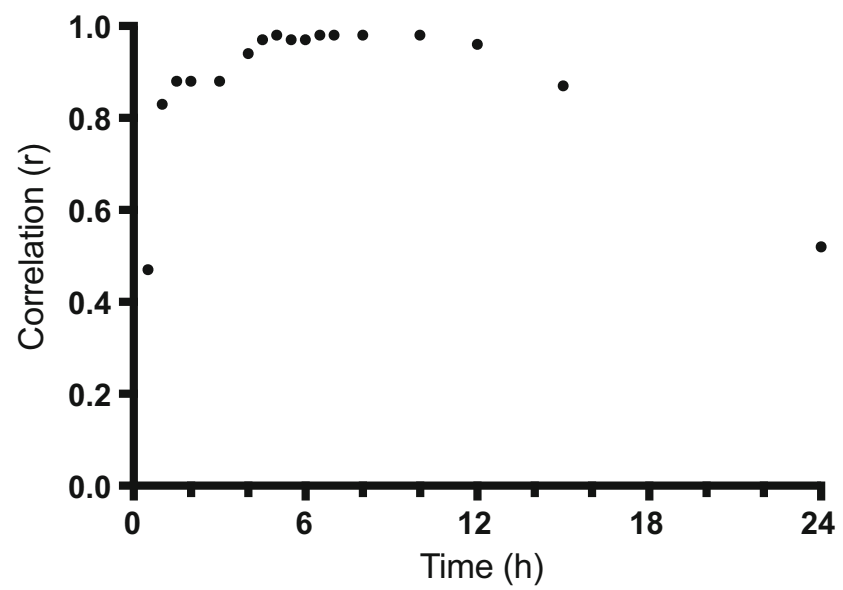

Fig. 3 Pearson correlation coefficients $(r)$ between single time point plasma d-RA/d-MPH ratios versus $\mathrm{AUC}_{\mathrm{d}-\mathrm{RA}} / \mathrm{AUC}_{\mathrm{d}-\mathrm{MPH}}$ ratios $(y$-axis) in relation to the corresponding time point after drug intake ( $x$-axis)

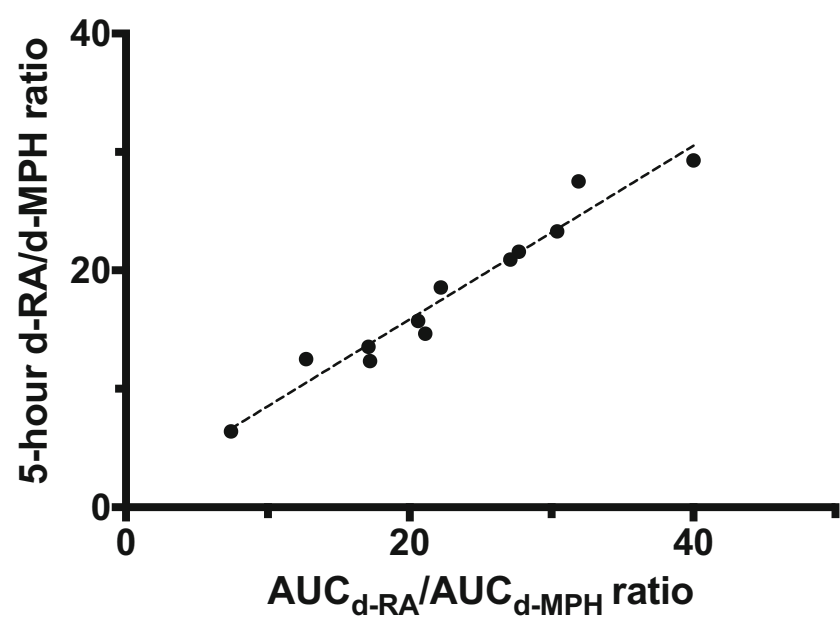

Fig. 4 Correlation of 5-hour d-RA/d-MPH ratios in plasma versus $\mathrm{AUC}_{\mathrm{d}-}$ $\mathrm{RA} / \mathrm{AUC}_{\mathrm{d}-\mathrm{MPH}}$ ratios. $r=0.98$

required for AUC determination would be more practical and inexpensive in clinical practice. The d-RA/d-MPH ratios at $5,6.5,7,8$, and $10 \mathrm{~h}$ after dose $(20 \mathrm{mg}$, Ritalin $\left({ }^{\circledR}\right)$ have the highest correlation with the AUC ratio $(r=0.98)$, Fig 3. However, the d-RA/d-MPH ratios at 1.5 , 2 , and $3 \mathrm{~h}$ might also be possible to use, as the correlation $(r=0.88)$ is reasonably high, and it would be favorable to use a single plasma sample for both TDM and determination of the metabolic ratio, as a measure of individual metabolic activity. Sampling closer to dosing may also be more convenient for the patients. The agreement between the two methods (AUC ratios and single-point measurements of metabolite/parent drug) needs to be further studied to assess which ratios and what timepoints would be most relevant for prediction of CES1 activity [30].

Interestingly, one female subject in our study had distinctly deviating pharmacokinetics of both enantiomers of

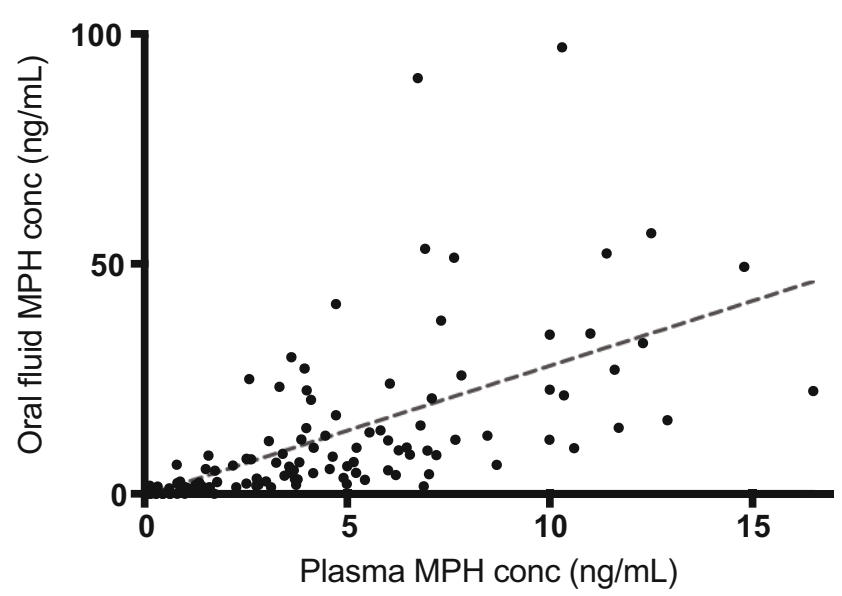

Fig. 5 Scatter plot of oral fluid dl-threo-methylphenidate concentrations in relation to the corresponding plasma concentration (sum of d- and 1$\mathrm{MPH})$. Linear regression. $y=2.817 \times-0.6341 ; r=0.61, N=103.17$ data points were excluded due to oral fluid concentration values below limit of quantification 
methylphenidate relative to the other eleven study subjects. She had the highest AUC of $d$-threo-methylphenidate, together with unexpectedly high concentrations of l-threo-methylphenidate. Moreover, her $\mathrm{AUC}_{\mathrm{d}-\mathrm{RA}} / \mathrm{AUC}_{\mathrm{d}-\mathrm{MPH}}$ ratio was the lowest of all the subjects studied. A possible explanation might be a low CES1 activity in this subject, resulting in higher bioavailability of l-threo-methylphenidate.

dl-threo-Methylphenidate is a weak base, with a low molecular mass (233 Da) and low plasma protein binding and crosses the membrane between blood and saliva in its ionized form via passive diffusion. As saliva is more acidic than blood, ion trapping is likely to occur, resulting in higher $d l$-threo-methylphenidate concentrations in saliva compared with blood [31]. In a study by Marchei et al. [32] in eight healthy volunteers, methylphenidate concentrations in oral fluid correlated with those in plasma ( $r=$ 0.79 ). In our study, we found a slightly lower correlation between methylphenidate concentrations in oral fluid and plasma $(r=0.61)$. Our data are in line with studies in patients showing three-to-four-fold higher methylphenidate concentrations in oral fluid than in blood, while the opposite was true for ritalinic acid [33, 34]. The present study indicates that oral fluid may serve as an alternative non-invasive matrix to assess medication adherence, as the oral fluid concentrations of dl-threo-methylphenidate in our study roughly reflected $d$-threo-methylphenidate concentrations in plasma.

dl-threo-Methylphenidate was detected in exhaled breath up to $24 \mathrm{~h}$ (last sampling point) in only five of twelve subjects. Exhaled breath may be useful for assessing whether a recent intake of methylphenidate has occurred, as methylphenidate was detected in $87 \%$ of the exhaled breath samples. Fluctuations in the amount of aerosol particles in the exhaled breath collected with the sampling device might explain the weak correlation between methylphenidate concentrations in plasma and exhaled breath [35]. The use of exhaled breath to assess medication adherence warrants further studies in patients, as the results may be different at the higher doses of methylphenidate used clinically.

Women tended to exhibit higher methylphenidate plasma AUC compared with men, but the difference did not reach statistical significance. Contradictory findings with lower bioavailability of methylphenidate in women compared with men have been reported [36]. It is important to emphasize that the numbers of male and female subjects studied in this respect are low. In a study in 19 subjects, men had on average 30\% lower methylphenidate dose $(\mathrm{mg} / \mathrm{kg})$ than women, without differences in average AUC [37]. In a population pharmacokinetic study in children $(N=273)$, the clearance and half-life were similar in boys and girls [38]. Further studies are required to investigate possible differences in the pharmacokinetics between men and women treated with methylphenidate.
The current study has some limitations. The small sample size precludes the proper analysis of sex differences. The dose of methylphenidate used may be too low to evaluate the feasibility of alternative matrices for monitoring medication adherence, as the doses used for patients with ADHD are much higher, especially in adults with comorbid substance use disorder. In conclusion, we report for the first time the simultaneous pharmacokinetic analysis of $d l$ threo-methylphenidate and its metabolite $\mathrm{dl}$-threo-ritalinic acid in oral fluid and exhaled breath in comparison to plasma. Our study highlighted the importance of chirality and the enantioselective determination of methylphenidate and ritalinic acid in plasma for TDM purposes. A single-point measurement of the d-RA/d-MPH ratio as a marker of methylphenidate metabolism was also investigated. Larger cohorts with different methylphenidate formulations and different doses, preferably genotyped for CES1, are required to assess which metabolic ratios would be most relevant in clinical practice.

Acknowledgments Open access funding provided by Karolinska Institute. We thank RN Marta Stenberg, MDs Georgios Panagiotidis, and Ludvig Petersson for excellent assistance during the study and Christoffer Tavic, biomedical laboratory scientist, for bioanalysis.

Author contributions Arvidsson, Dahl, Rosenborg, and Nordin designed the clinical trial, which was performed by Arvidsson and Nordin. Ackehed developed the method for the enantioselective analysis of methylphenidate and ritalinic acid in plasma and was responsible for the analysis of the enantiomers in plasma. Ackehed, Arvidsson, Dahl, Beck, and Rosenborg analyzed the data. All authors were involved in drafting and reviewing the manuscript and approved the final version.

Funding information The work was funded by the Stockholm County Council (ALF 20150043, 20180534), the Swedish Brain Foundation and by the Swedish Research Council (2015-02836).

\section{Compliance with ethical standards}

Conflict of interest The authors declare that they have no conflict of interest.

Open Access This article is distributed under the terms of the Creative Commons Attribution 4.0 International License (http:// creativecommons.org/licenses/by/4.0/), which permits unrestricted use, distribution, and reproduction in any medium, provided you give appropriate credit to the original author(s) and the source, provide a link to the Creative Commons license, and indicate if changes were made.

\section{References}

1. Simon V, Czobor P, Balint S, Meszaros A, Bitter I (2009) Prevalence and correlates of adult attention-deficit hyperactivity disorder: meta-analysis. Br J Psychiatry 194(3):204-211 
2. Polanczyk G, de Lima MS, Horta BL, Biederman J, Rohde LA (2007) The worldwide prevalence of ADHD: a systematic review and metaregression analysis. Am J Psychiatry 164(6):942-948

3. Pliszka SR (2007) Pharmacologic treatment of attention-deficit/hyperactivity disorder: efficacy, safety and mechanisms of action. Neuropsychol Rev 17(1):61-72

4. Raman SR, Man KKC, Bahmanyar S, Berard A, Bilder S, Boukhris $\mathrm{T}$ et al (2018) Trends in attention-deficit hyperactivity disorder medication use: a retrospective observational study using population-based databases. Lancet Psychiatry 5(10):824-835

5. Heal DJ, Pierce DM (2006) Methylphenidate and its isomers: their role in the treatment of attention-deficit hyperactivity disorder using a transdermal delivery system. CNS Drugs 20(9):713-738

6. Kimko HC, Cross JT, Abernethy DR (1999) Pharmacokinetics and clinical effectiveness of methylphenidate. Clin Pharmacokinet 37(6):457-470

7. Frolich J, Banaschewski T, Dopfner M, Gortz-Dorten A (2014) An evaluation of the pharmacokinetics of methylphenidate for the treatment of attention-deficit/ hyperactivity disorder. Expert Opin Drug Metab Toxicol 10(8):1169-1183

8. Srinivas NR, Hubbard JW, Korchinski ED, Midha KK (1993) Enantioselective pharmacokinetics of dl-threo-methylphenidate in humans. Pharm Res 10(1):14-21

9. Sun Z, Murry DJ, Sanghani SP, Davis WI, Kedishvili NY, Zou Q et al (2004) Methylphenidate is stereoselectively hydrolyzed by human carboxylesterase CES1A1. J Pharmacol Exp Ther 310(2): 469-476

10. Zhu HJ, Patrick KS, Yuan HJ, Wang JS, Donovan JL, DeVane CL et al (2008) Two CES1 gene mutations lead to dysfunctional carboxylesterase 1 activity in man: clinical significance and molecular basis. Am J Hum Genet 82(6):1241-1248

11. Faraj BA, Israili ZH, Perel JM, Jenkins ML, Holtzman SG, Cucinell SA, Dayton PG (1974) Metabolism and disposition of methylphenidate-14C: studies in man and animals. J Pharmacol Exp Ther 191(3):535-547

12. Schoretsanitis G, Paulzen M, Unterecker S, Schwarz M, Conca A, Zernig G, Gründer G, Haen E, Baumann P, Bergemann N, Clement HW, Domschke K, Eckermann G, Egberts K, Gerlach M, Greiner C, Havemann-Reinecke U, Hefner G, Helmer R, Janssen G, Jaquenoud-Sirot E, Laux G, Messer T, Mössner R, Müller MJ, Pfuhlmann B, Riederer P, Saria A, Schoppek B, Silva Gracia M, Stegmann B, Steimer W, Stingl JC, Uhr M, Ulrich S, Waschgler R, Zurek G, Hiemke C (2018) TDM in psychiatry and neurology: a comprehensive summary of the consensus guidelines for therapeutic drug monitoring in neuropsychopharmacology, update 2017; a tool for clinicians. World J Biol Psychiatry 19(3):162-174

13. Patrick KS, Caldwell RW, Ferris RM, Breese GR (1987) Pharmacology of the enantiomers of threo-methylphenidate. J Pharmacol Exp Ther 241(1):152-158

14. Markowitz JS, Patrick KS (2008) Differential pharmacokinetics and pharmacodynamics of methylphenidate enantiomers: does chirality matter? J Clin Psychopharmacol 28(3 Suppl 2):S54-S61

15. Mannheimer B, Haslemo T, Lindh JD, Eliasson E, Molden E (2016) Risperidone and venlafaxine metabolic ratios strongly predict a CYP2D6 poor metabolizing genotype. Ther Drug Monit 38(1): 127-134

16. Scordo MG, Spina E, Facciola G, Avenoso A, Johansson I, Dahl ML (1999) Cytochrome P450 2D6 genotype and steady state plasma levels of risperidone and 9-hydroxyrisperidone. Psychopharmacology 147(3):300-305

17. Safren SA, Duran P, Yovel I, Perlman CA, Sprich S (2007) Medication adherence in psychopharmacologically treated adults with ADHD. J Atten Disord 10(3):257-260

18. Eliasson E, Lindh JD, Malmstrom RE, Beck O, Dahl ML (2013) Therapeutic drug monitoring for tomorrow. Eur J Clin Pharmacol 69(Suppl 1):25-32
19. Hiemke C, Bergemann N, Clement HW, Conca A, Deckert J, Domschke K, Eckermann G, Egberts K, Gerlach M, Greiner C, Gründer G, Haen E, Havemann-Reinecke U, Hefner G, Helmer R, Janssen G, Jaquenoud E, Laux G, Messer T, Mössner R, Müller MJ, Paulzen M, Pfuhlmann B, Riederer P, Saria A, Schoppek B, Schoretsanitis G, Schwarz M, Gracia MS, Stegmann B, Steimer W, Stingl JC, Uhr M, Ulrich S, Unterecker S, Waschgler R, Zernig G, Zurek G, Baumann P (2018) Consensus guidelines for therapeutic drug monitoring in neuropsychopharmacology: update 2017. Pharmacopsychiatry. 51(1-02):e1

20. Gallardo E, Queiroz JA (2008) The role of alternative specimens in toxicological analysis. Biomed Chromatogr 22(8):795-821

21. Arvidsson M, Ullah S, Franck J, Dahl ML, Beck O (2019) Drug abuse screening with exhaled breath and oral fluid in adults with substance use disorder. Drug Test Anal 11(1):27-32

22. Drummer OH (2006) Drug testing in oral fluid. Clin Biochem Rev 27(3):147-159

23. Beck O, Sandqvist S, Eriksen P, Franck J, Palmskog G (2010) Method for determination of methadone in exhaled breath collected from subjects undergoing methadone maintenance treatment. J Chromatogr B Anal Technol Biomed Life Sci 878(24):2255-2259

24. European Commission, Guidance documents applying to clinical trials in EudraLex $10 \mathrm{https}: / /$ ce.europa.eu/health/documents/ eudralex/vol-10_sv. Accessed 1 May 2019

25. Skoglund C, Hermansson U, Beck O (2015) Clinical trial of a new technique for drugs of abuse testing: a new possible sampling technique. J Subst Abus Treat 48(1):132-136

26. Beck O, Stephanson N, Sandqvist S, Franck J (2013) Detection of drugs of abuse in exhaled breath using a device for rapid collection: comparison with plasma, urine and self-reporting in 47 drug users. J Breath Res 7(2):026006

27. Ullah S, Sandqvist S, Beck O (2018) A liquid chromatography and tandem mass spectrometry method to determine 28 non-volatile drugs of abuse in exhaled breath. J Pharm Biomed Anal 148:251258

28. Zhang Y, Huo M, Zhou J, Xie S (2010) PKSolver: An add-in program for pharmacokinetic and pharmacodynamic data analysis in Microsoft Excel. Comput Methods Prog Biomed 99(3):306-314

29. Thomsen R, Rasmussen HB, Linnet K, Consortium I (2012) Enantioselective determination of methylphenidate and ritalinic acid in whole blood from forensic cases using automated solid-phase extraction and liquid chromatography-tandem mass spectrometry. J Anal Toxicol 36(8):560-568

30. Stage C, Dalhoff K, Rasmussen HB, Guski LS, Thomsen R, Bjerre D et al (2019) The impact of human CES1 genetic variation on enzyme activity assessed by ritalinic acid/methylphenidate ratios. Basic Clin Pharmacol Toxicol 125(1):54-61

31. Secilir A, Schrier L, Bijleveld YA, Toersche JH, Jorjani S, Burggraaf J et al (2013) Determination of methylphenidate in plasma and saliva by liquid chromatography/tandem mass spectrometry. J Chromatogr B Anal Technol Biomed Life Sci 923-924:22-28

32. Marchei E, Farre M, Pardo R, Garcia-Algar O, Pellegrini M, Pacifici R et al (2010) Correlation between methylphenidate and ritalinic acid concentrations in oral fluid and plasma. Clin Chem 56(4):585-592

33. Josefsson M, Rydberg I (2011) Determination of methylphenidate and ritalinic acid in blood, plasma and oral fluid from adolescents and adults using protein precipitation and liquid chromatography tandem mass spectrometry-a method applied on clinical and forensic investigations. J Pharm Biomed Anal 55(5):1050-1059

34. Cherma MD, Josefsson M, Rydberg I, Woxler P, Trygg T, Hollertz O et al (2017) Methylphenidate for treating ADHD: a naturalistic clinical study of methylphenidate blood concentrations in children and adults with optimized dosage. Eur J Drug Metab Pharmacokinet 42(2):295-307 
35. Almstrand AC, Ljungstrom E, Lausmaa J, Bake B, Sjovall P, Olin AC (2009) Airway monitoring by collection and mass spectrometric analysis of exhaled particles. Anal Chem 81(2):662-668

36. Patrick KS, Straughn AB, Reeves OT 3rd, Bernstein H, Bell GH, Anderson ER et al (2013) Differential influences of ethanol on early exposure to racemic methylphenidate compared with dexmethylphenidate in humans. Drug Metab Dispos 41(1):197205

37. Markowitz JS, Straughn AB, Patrick KS (2003) Advances in the pharmacotherapy of attention-deficit-hyperactivity disorder: focus on methylphenidate formulations. Pharmacotherapy. 23(10):12811299

38. Shader RI, Harmatz JS, Oesterheld JR, Parmelee DX, Sallee FR, Greenblatt DJ (1999) Population pharmacokinetics of methylphenidate in children with attention-deficit hyperactivity disorder. J Clin Pharmacol 39(8):775-785

Publisher's note Springer Nature remains neutral with regard to jurisdictional claims in published maps and institutional affiliations. 